\title{
SEXUALIDADE, AIDS E DROGAS: UM RELATO DE INTERVENÇÃO COM ADOLESCENTES
}

\author{
SEXUALITY, AIDS AND DRUGS: AN INTERVENTION \\ REPORT WITH ADOLESCENTS
}

\author{
Renata Maria Dotta \\ Paola Biasoli Alves \\ Silvia Helena Koller \\ Raquel Cardoso Brito ${ }^{1}$
}

DOTTA, R. M.; ALVES, P. B.; KOLLER, S. H.; BRITO, R. C. Sexualidade, AlDs e drogas: um relato de intervenção com adolescentes. Rev. Bras. Cresc. Desenv. Hum., São Paulo, 10 (2), 2000.

Resumo: Este artigo apresenta o Programa de Intervenção em Sexualidade, AIDS e Drogas (PISAD) da Universidade Federal do Rio Grande do Sul e um exemplo de atividade de intervenção com adolescentes em situação de risco realizado por ele. São apresentados os pressupostos da Abordagem Ecológica do Desenvolvimento Humano e da Psicologia Comunitária (teorias que apoiam o trabalho do PISAD) e do desenvolvimento adolescente, ressaltando as questões de comportamentos de risco para Sexualidade saudável, HIV/AIDS e drogas. Na intervenção com os adolescentes foi realizado um levantamento de necessidades no inicio e aplicado um instrumento de sentenças incompletas no final, centrados nas temáticas do PISAD. As informações obtidas foram analisadas e mostram a existência de dúvidas com relação à transmissão e prevenção do HIV, a crença na necessidade de urna rede de apoio sócio-emocional efetiva para a criação e promoção de estratégias que estimulem comportamentos saudáveis e uma associação clara entre as experiências vividas no cotidiano e questões do desenvolvimento adolescente, como autoestima, identidade, grupo de amigos e vida sexual.

Palavras-chave: sexualidade; HIV/AIDs; drogas; adolescente; intervenção.

\section{OBJETIVO}

Este artigo tem por objetivo apresentar um modelo de programa de intervenção em Sexualidade, AIDS e Drogas, inserido no contexto de extensão universitária e voltado para a promoçao e implementação de trabalhos sobre estes temas em diferentes comunidades. Explicita um processo de intervenção pontual desenvolvido por este programa, atendendo adolescentes de nivel sócioeconômico baixo. Desta intervenção são apresentados os dados do levantamento de necessidades e da avaliação do processo. Os modelos teóricos metodológicos que sustentam a criação e a efetivação do programa de intervenção são descritas e discutidos, assim como, os aspectos evolutivos relevantes da fase de desenvolvimento atendida.

\section{INTRODUÇÃO}

Nos últimos anos, o elevado índice de gravidez na adolescência, o crescente uso indevido de drogas e a disseminação do Vírus da Imuno

1 Universidade Federal do Rio Grande do Sul, instituto de Psicologia, Centro de Estudos Psicológicos sobre Meninos e Meninas de Rua.

Endereço para Correspondência: Rua Ramiro Barcelos, 2600, sala 104, 90035-003. Porto Alegre, Brasil. Fone: 51 316-5150 Fax: 51.330-4797. E-mail: paolabia@yahoo.com 
Deficiênica Humana (HIV) e suas conseqüências mobilizaram diversas camadas da sociedade. Hoje busca-se alternativas de sensibilizarão, conscientização e prevenção direcionados às temáticas de sexualidade, AIDS e Drogas, entendendo-se estes como procedimentos associados a melhoria da qualidade de vida e viabilizadores do exercício da cidadania.

A participação da Universidade no processo de produção e divulgação de conhecimento referentes a estes temas é fundamental. Dentro da Universidade Federal do Rio Grande do Sul (UFRGS), inserido na proposta de pesquisa, ensino e extensão do Centro de Estudos Psicológicos sobre Meninos e Meninas de Rua (CEP-RUA) existe, desde 1997, o Programa de Intervenção em Sexualidade, AIDS e Drogas (PISAD). Este programa busca desenvolver um trabalho qualificado junto à comunidade, capacitando estudantes e profissionais das áreas da saúde e da educação para intervir em diversas situações ligadas aos temas Sexualidade, AIDS e Drogas e sistematizar a produção do conhecimento obtido.

Como base para o trabalho do PISAD são adotados os referenciais teórico-metodológicos da Psicologia Comunitária e da Abordagem Ecológica do Desenvolvimento Humano (BRONFENBRENNER, 1979/1996, 1993, 1995; BRONFENBRENNER \& MORRIS, 1998). A primeira referência evidencia a necessidade do trabalho comunitário ser compreendido e vivenciado enquanto um processo de construção e apropriação do conhecimento, realizado na interação entre profissionais, técnicos e a comunidade envolvida. Este processo compreende o levantamento das necessidades do grupo atendido, a busca de parcerias e de soluções adequadas para cada situação, estimulando a autonomia comunitária e a conscientização através da participação (DUFFY \& WONG, 1996; LEVINE \& PERKINS, 1987; ORFORD, 1992). O segundo referencial apresenta a idéia do desenvolvimento humano enquanto um processo de interação dinâmica e integrada entre pessoa-ambiente, com ênfase nos aspectos evolutivos saudáveis (BRONFENBRENNER, 1995; BRONFENBRENNER \& MORRIS, 1998). É importante, na Abordagem Ecológica do Desenvolvimento Humano, analisar características de Tempo, Pessoa, Processo e Contexto que envolvem o crescimento de cada pessoa. O momento histórico e a fase de desenvolvimento vividos, os pares de interação, a significação das experiências e os valores e crenças presentes na vida cotidiana de cada membro da comunidade são exemplos da importância da interação pessoa-ambiente para esta abordagem (BRONFENBRENNER \& MORRIS, 1998).
Nos trabalhos realizados pelo PISAD, a aplicação dos referenciais teóricometodológicos encontram-se sistematizados e são aplicados nas seguintes atividades: A) cursos de capacitação e de formação continuada para estudantes universitários, profissionais da saúde e educação, técnicos e agentes comunitários que já estejam envolvidos ou desejam se envolver em projetos de prevenção ligados aos temas referidos. Nas capacitações, as técnicas utilizadas durante os cursos são passadas para o grupo, assim como o material de apoio didático, criando-se um espaço de multiplicação do conhecimento; B) assessoria a empresas e instituições públicas e privadas que desejam promover e/ou implementar programas de intervenção e prevenção dentro deste contexto; C) intervenções pontuais realizadas em escolas, empresas e instituições, atendendo adolescentes e adultos, com o objetivo de informar e sensibilizar os participantes para questões como a transmissão do HIV, contracepção, relações de gênero, abuso de substâncias psicoativas e os riscos para o desenvolvimento, D) levantamento de necessidades dos grupos atendidos, com o objetivo de direcionar as intervenções e contribuir para a construção do conhecimento na área.

Em cada intervenção proposta, procura-se manter as seguintes regras: 1 ) os trabalhos são extensos no tempo (cada atividade tem duração mínima de 10 horas); 2) são atendidos no máximo 45 pessoas em cada atividade; e, 3) privilegia-se o uso de dinâmicas pedagógicas e técnicas de sensibilização para a apresentação e discussão dos temas ligados à Sexualidade, AIDS e Drogas. O procedimento básico seguido dentro de cada atividade ‘ consiste em que cada grupo tenha suas necessidades e expectativas levantadas e se co-responsabilize pelo processo de aprendizagem a ser iniciado. Dentro da multiplicidade de subtemas possíveis originados dos grandes temas principais, valoriza-se aqueles que permitem discussões sobre crenças, preconceitos, expectativas e valores, sempre apoiados por reflexões sobre cada fase do desenvolvimento humano, enfatizando-se os aspectos sócio-emocionais envolvidos.

Para exemplificar a atuação do PISAD, a seguir sèrá apresentado um processo de intervenção com adolescentes realizado no segundo semestre de 1998. Inicialmente, é necessário apresentar e discutir aspectos relevantes do desenvolvimento sócio-emocional na adolescência e suas implicações em intervenções sobre Sexualidade, AIDS e Drogas. 


\section{ADOLESCÊNCIA: DESENVOLVIMENTO, SEXUALIDADE, AIDS E DROGAS}

A adolescência é uma fase do desenvolvimento humano considerada como muito conturbada, caracterizando-se como período de profundas transformações, quando são revistos e consolidados valores e atitudes. Neste momento, geralmente, o jovem se sente adulto e se entristece quando é tratado como criança. Ao mesmo tempo, fica angustiado quando se sente criança e lhe são exigidos comportamentos adultos (STEINBERG, 1999).

Ao lado das dúvidas que estão em torno da busca de uma identidade própria, e em conseqüência de todas as mudanças biológicas, psicológicas, emocionais e sociais, o adolescente enfrenta, também, a dificuldade em lidar com a própria sexualidade. Deste modo, sofre todas as pressões sociais e morais advindas da igreja, da família e da comunidade e, por outro lado, a pressão da média e dos amigos recebendo, de ambos os lados, informações variadas e, muitas vezes, paradoxais. Ao receber este conjunto de idéias, algumas vezes contraditórias ou causadoras de dúvidas, o jovem não encontra espaço para debater suas ansiedades, refletir sobre seus valores e conflitos. Isto contribui para que o adolescente não consiga viver sua sexualidade sem medo e/ ou culpa, expondo-se a comportamentos sexuais de risco (RIBEIRO, 1990; STEINBERG, 1999).

Os processos de intervenção voltados para o trabalho da Sexualidade com adolescentes devem ser guiados pela busca da desmistificação dos estereótipos sexuais através de uma relação de troca e não de informação-recepção unilateral. É relevante que o adolescente tenha acesso às informações corretas; porém, quando se trata de crenças e valores, sabe-se que apenas a informação não é o bastante. Há a necessidade de se trabalhar mitos e tabus que envolvem o tema da sexualidade, valorizando uma prática saudável e consciente (BERNARDIS, 1985; RIBEIRO, 1990).

Aprofundando estas idéias, o trabalho com adolescentes implica a conjunção de vários fatores que explicam o desenvolvimento sexual como: saúde reprodutiva, relacionamento interpessoal, afetividade, auto-estima, imagem corporal e relações de gênero. Em um sentido amplo, o entendimento da Sexualidade ocorre através do inter-relacionamento de diversos aspectos intelectuais, afetivos, familiares e sociais, dentro de um contexto ético e moral, das leis culturais e valores sociais do meio em que vivem os jovens (GOWÊA, 1995; SAYÃO, 1997).

CORCORAN, MILLER \& BULTAM (1997) apontam a necessidade de trabalhar com crenças e mitos erróneos acerca da contracepção. MURPHI \& BOGGER (1998) demostram que as atitudes e crenças a respeito da eficácia e da representação social que giram em torno do uso de preservativos são os maiores determinantes de seu uso. MACEDO \& SOUZA (1996) encontraram em sua pesquisa com adolescentes a crença de que "meninas devem ser mais cuidadosas" com a contracepção. Estes autores, de modo geral, afirmam que todo o universo das relações de gênero é a variável fundamental no trabalho com adolescentes.

No trabalho sobre sexualidade com os adolescentes, aspectos relativos a Doenças Sexualmente Transmissíveis (DSTs) e a Síndrome de Imuno Deficiência Adquirida (SIDA/AIDS) também não devem ser negligenciados. BOYER, SHAFER \& TSCHAN (1997) enfatizam que para discutir e refletir sobre estes temas, os adolescentes necessitam mais do que a informação correta sobre os riscos e a prevenção da contaminação. Precisam, também, exercitar e fortalecer habilidades para resistir a pressões grupais, para negociar o uso de preservativos e para refletir sobre as possíveis conseqüências de seus atos.

DICLEMENTE (1998) afirma que intervenções que enfatizam o sexo seguro são significativamente mais efetivas na utilização posterior de preservativos no comportamento de adolescentes, do que as intervenções baseadas na abstinência. Os resultados demonstrados por GREEMLEY \& LEE (1997) sugerem que intervenções designadas a enfatizar o uso consistente e correto do preservativo são os meios mais eficazes para reduzir o número de gravidez indesejada e de DSTs.

Um trabalho que envolva todas as questões da adolescência deve, também, abordar questões relativas à prevenção do abuso de drogas. Estudos indicam que comportamentos de risco associados com a fase da adolescência tais como gravidez na adolescência, contaminação por DSTs, AIDS ou problemas relacionados a delitos, homicídios ou suicídios estão diretamente ligados ou são agravados pelo uso indevido de drogas (BRUNER \& FISHMAN, 1998).

Autores que estudam sobre abuso de drogas, fatores de proteção e estratégias de prevenção enfatizam a necessidade de trabalhos de intervenção que visem à conscientização e ao oferecimento de estratégias que ajudem o jovem a resistir à pressão grupal (BRUNER \& FISHMAN, 1998; KLEIN, 1997; WHEELER, 1997). Também é importante o resgate e a utilização dos meios de proteção oferecidos pela rede de apoio social, como elementos fundamentais na prevenção ao abuso de drogas (BRITO, 1999). A complexidade dos temas aqui descritos, ao lado 
de sua relevância, apresentam a necessidade de trabalhos direcionados à prevenção da exposição de adolescentes a comportamentos de risco. Neste sentido, atender-se-ia não somente uma demanda cada vez mais elevada, mas a um direito que deveria ser exigido por esses jovens.

No exemplo de intervenção pontual, descrito a seguir, o grupo de adolescentes atendidos encontrava-se dentro de uma situação especial de desenvolvimento. Além das características típicas desta fase, o nível sócio-econômico baixo é desfavorável e contribui para que os fatores de risco se intensifiquem. De acordo com a literatura nas áreas da Psicologia e Psicopatologia do Desenvolvimento, a presença de miséria econômica e/ ou afetiva vincula-se a desvantagens na evolução física, cognitiva e sócio-emocional dos seres humanos, podendo aumentar a freqüência de comportamentos de risco, entre eles a prática sexual desprotegida, o compartilhamento de seringas, e o uso indevido de drogas (GARMEZY \& MASTEN, 1994; RUTTER, 1993).

\section{O PROCESSO DE INTERVENÇÃO}

O processo de intervenção com os adolescentes foi proposto por uma empresa pública que, naquele momento, oferecia para os participantes um programa que abrangia treinamento profissional e apoio sócio educativo, com o objetivo de implementar a qualidade de vida e estimular a capacitação para o trabalho. O contato com o PISAD foi viabilizado pela estagiária do Serviço Social que conhecia as propostas do programa e procurava instrumentos adequados para auxiliar na efetivação dos objetivos sócio-educativos da empresa, enfatizando a promoção da saúde dos adolescentes.

Foram atendidos os quarenta e dois adolescentes que participavam deste programa, sendo dez do sexo feminino e trinta e dois do sexo masculino, de idades entre quatorze e dezessete anos, estudantes do Ensino Fundamental (terceira a oitava série). É importante dizer que a freqüência escolar era uma variável determinante para inserção do adolescente no programa da empresa. Os adolescentes estavam distribuídos em dois turnos de trabalho (manhã e tarde), no horário inverso do escolar. As atividades do PISAD foram realizadas dentro do horário de trabalho, e o procedimento adotado foi o mesmo para cada grupo.

No primeiro momento do processo de intervenção foi feito o levantamento de dúvidas dos adolescentes sobre Sexualidade, AIDS e Drogas através da técnica "Caixinha de Dúvidas"
(ALVES, BRITO \& DOTTA, 1997). O objetivo desta técnica foi nortear a elaboração da intervenção e a escolha adequada das dinâmicas pedagógicas e técnicas de sensibilização. Esta dinâmica consiste no oferecimento de papel e caneta para os participantes e a coordenadora solicita que todos escrevam suas principais dúvidas sobre cada assunto. Os participantes não precisavam se identificar. As dúvidas são colocadas em uma Caixinha e, posteriormente, lidas e discutidas pelas coordenadoras para organizar a intervenção.

Baseado neste levantamento de necessidades, o trabalho de intervenção propriamente dito consistiu na adaptação das dinâmicas e técnicas de sensihilizacão à realidade do grupo presente (vivência cotidiana de situações de risco e participação em programa profissionalizante e sócioeducativo), oferecendo, dentro de cada atividade, espaço para reflexão, discussão e avaliação do exercício realizado e suas implicações. Foram utilizadas, cronologicamente, as seguintes técnicas: Crachás (apresentação e integração do grupo); Bilhete nas Costas (a importância da comunicação nos relacionamentos interpessoais); Caixinha de Dúvidas (levantamento das necessidades); História de João e Thaís (discussão de relações de gênero e contracepção); Batata Quente (respostas oferecidas pelo próprio grupo às perguntas contidas na Caixinha de Dúvidas); Autógrafos (visualização da cadeia de transmissão do HIV); O que Sabemos sobre AIDS (exercício informativo sobre formas de transmissão e prevenção do HIV); A História de Marcelo (técnica de dramatização sobre a vida de jovens HIV positivos); Vestindo a Camisinha (técnica lúdica e informativa sobre o uso correto de preservativo) (ALVES, BRITO \& DOTTA, 1997).

No término da intervenção, que teve duração aproximada de dez horas com cada grupo, foi solicitado que os adolescentes respondessem ao Jogo de Sentenças Incompletas sobre Sexualidade, AIDS e Drogas (ALVES, BRITO \& DOTTA, 1998). Este é um instrumento que contém oito frases com final aberto e deve ser respondido por escrito, sem preocupação com a formalização das respostas. Sua aplicação teve o objetivo de reunir informações sobre a opinião dos adolescentes acerca das temáticas trabalhadas, bem como oferecer subsídios para a continuidade do processo sócio-educativo, nesta área, pela própria instituição nos pontos que se considerassem relevantes.

A leitura das dúvidas trazidas pelos adolescentes (recolhidas através da Caixinha de Dúvidas), além de servir para a elaboração do programa de intervenção pontual, podem ser apresentadas e discutidas a partir da Tabela 1. 
Tabela 1: Freqüência de Dúvidas sobre Sexualidade, AIDS e Drogas.

\begin{tabular}{lccc}
\hline Agrupamento & Informativa & Motivacional & Valorativa \\
\hline Drogas & 5 & 4 & 0 \\
AIDS & 20 & 0 & 7 \\
Sexualidade & 6 & 0 & 2 \\
\hline
\end{tabular}

Na Tabela 1 as perguntas motivacionais referem-se a comportamentos, motivos e intenções que podem levar o adolescente a usar drogas; as informativas referem-se a dúvidas gerais sobre os três temas (como usar métodos contraceptivos, quais os meios de transmissão do HIV, o que é o crack, etc.) e as valorativas referem-se a dúvidas geradas por crenças e/ou valores que envolvem AIDS e sexualidade.

Os resultados mostram que as dúvidas com relação a informações sobre meios de transmissão e prevenção do HIV/AIDS foram as mais freqüentes. As questões referentes a valores e crenças ligadas à síndrome apareceram em segundo lugar. No que se refere ao tema Drogas, as dúvidas referiam-se a informação sobre danos causados e motivos que levariam as pessoas a consumi-las. Nas questões referentes a Sexualidade, a maior freqüência de dúvidas esteve relacionada a informações sobre contracepção, valores e preconceitos. Os dados aqui descritas foram obtidos a partir da leitura das dúvidas dos adolescentes e de sua contagem, possibilitando sua apresentação através de frequência simples.

As respostas dos adolescentes ao Jogo de Sentenças Incompletas foram analisadas através de um modelo quantitativo-interpretativo, como proposto por BIASOLI ALVES (1988). Fez-se a leitura das sentenças e foram identificados temas comuns presentes em cada uma das respostas. A partir da discussão deste conteúdo foram criadas 44 categorias: vinte e sete para Drogas (sentenças 1, 2, 3); oito para HIV/AIDS (sentenças 4 e 5) e nove para Sexualidade (sentenças 6, 7, 8). A Tabela 2 apresenta uma sistematização destes dados, mostrando as frequências das categorias mais representativas encontradas.

A análise dos resultados das sentenças incompletas, conforme apresentado na Tabela 2, revela que os adolescentes desta amostra acreditam que os principais fatores que levam ao uso de drogas são aqueles ligados aos problemas ou desavenças familiares, amizades, curiosidade e insegurança emocional. Esses jovens atribuem o não uso de drogas a razões tais como: "acredita de que tem informação suficiente”, "gostar de si próprio", "ser inteligente/consciente" e "estar ciente dos prejuízos que trazem à saúde”. Na sentença 3 (O usuário deixará de usar drogas quando...) podese verificar a crença da impossibilidade de parar com o uso de drogas por esforço próprio, apre-

Tabela 2: Jogo de Sentenças Incompletas e Freqüência das Categorias mais Representadas ( $\mathrm{n}^{\circ}$ de respostas por categoria indicado entre parênteses)

\begin{tabular}{ll}
\multicolumn{1}{c}{ Sentenças Incompletas } & \multicolumn{1}{c}{ Categorias } \\
\hline 1. Os motivos que levam ao uso de drogas são ... & $\begin{array}{l}\text { Problemas/conflitos familiares (11), } \\
\text { Amizades (10), curiosidade (10) } \\
\text { Baixa auto estima/ insegurança afetiva (6) }\end{array}$ \\
2. Algumas pessoas não usam drogas porque .... & $\begin{array}{l}\text { Inforl Informação/ crença (11), alta auto estima (9) } \\
\text { Inteligente/consciente (9), prejuizos a saúde (7), } \\
\text { Rede de apoio afetiva (1) }\end{array}$ \\
3. O usuário deixará de usar drogas quando .... & $\begin{array}{l}\text { Impossibilidade de deixar de usar drogas (9), } \\
\text { Tratamento especializado (8) } \\
\text { Conscientização (8), rede de apoio efetiva (5) }\end{array}$ \\
4. O portador de HIV precisa de ... & $\begin{array}{l}\text { Rede de apoio social e afetiva (48), tratamento } \\
\text { especializado (9) } \\
\text { Disposição/ dever de ajudar (20), } \\
\text { Crenças sobre informação/transmissão e manifestação } \\
\text { do HIV (10), } \\
\text { Tratamento igualitário (8), preconceito (2) } \\
\text { Prevenida (21), inteligente/consciente (20), valoriza o } \\
\text { prazer (3), prostituta, malandra (2) } \\
\text { Prevenção (41), conscientização (9) } \\
\text { Doenças sexualmente transmissiveis (31) } \\
\text { Doenças passiveis de tratamento e prevenção (7) }\end{array}$ \\
\end{tabular}


sentando respostas como por exemplo: "quando proibirem", "quando estiver no fundo do poço" ou "quando morrer". Um número menor de respostas assinalam a importância da rede de apoio social e afetiva (amigos, famílias) como fonte efetiva de ajuda na interrupção do uso.

Nas sentenças referentes às questões ligadas ao HIV/AIDS nota-se que a totalidade das respostas dos adolescentes revelou a importância de uma rede de apoio social e afetiva das quais "ajuda de amigos", "apoio da família”, "solidariedade” foram as características "mais citadas. A categoria "disposição/dever de ajudar" se mostrou relevante frente ao relacionamento com pessoas portadoras do vírus HIV. Respostas associadas a preconceitos e crenças a respeito dos sintomas típicos de pessoas portadoras também foram apresentados, porém em menor número.

Na sentença 6 ("A mulher que carrega uma camisinha é...”) as categorias mais emergentes foram "prevenida" e "inteligente/consciente". Dentro da categoria "prostituta/malandra", apenas duas respostas foram apresentadas. Na sentença 7 ("Quando decido começar a transar devo...") todos os participantes assinalaram aimportância da prevenção, em especial, o uso da camisinha. Outras respostas tais como: "ter consciência de que quer aquilo mesmo", "fazer com segurança” ou "ter responsabilidade" foram citadas dentro da categoria “conscientização", porém em menor número. Na última sentença ("DSTs são...") as respostas evidenciaram que os jovens conhecem o significado dessa sigla e a importância de sua prevenção.

\section{DISCUSSÃO}

A apresentação do programa de intervenção (PISAD), o exemplo de intervenção pontual realizado com adolescentes, e as informações obtidas no levantamento de necessidades e nas respostas ao Jogo de Sentenças Incompletas proporcionam parâmetros relevantes para a discussão sobre a inter-relação entre teoria e trabalho técnico, quando o assunto é Adolescência, Sexualidade, AIDS e Drogas.

As características sócio-econômicas do grupo atendido delinearam um processo de intervenção particularizado, realizado a partir da adaptação das técnicas originais e do uso de uma linguagem específica, para a apresentação e discussão das informações. Foi importante trazer os temas de Sexualidade, AIDS e Drogas exemplificando-os no cotidiano dos adolescentes, valorizando sua sensibilização para as questões relativas a sua própria comunidade.
As dúvidas apresentadas no instrumento 1 (Caixinha de Dúvidas) mostraram a preocupação dos adolescentes em obter a informação correta sobre os temas propostos. Analisando este aspecto através dos pressupostos do desenvolvimento sócio-emocional desta fase, pode-se associar a grande freqüência de perguntas de caráter informativo com a necessidade do adolescente de confrontar opiniões e sistematizar o seu próprio conhecimento. Este dado revela a importância dos programas de intervenção oferecerem espaços tanto de sensibilização quanto de trocas de informação, valorizando a adolescência como uma fase privilegiada de construção da identidade, apoiada nas experiências comportamentais, cognitivas e afetivas. Estiveram presentes, ainda, nas perguntas elaboradas pelos adolescentes, as questões que envolviam crenças, valores e motivos associados a comportamentos sexuais, de abuso de drogas e do contágio com HIV. Este dado mostra a relação intrínseca entre pessoa ambiente no decorrer do desenvolvimento. Estão envolvidas características pessoais, interpessoais, e em um sentindo mais amplo, características sócio-histórico-culturais que se refletem em estereótipos, preconceitos, mitos e tabus perpetuados através de gerações.

Os dados obtidos através das sentenças incompletas revelaram a interação entre as experiências de vida destes adolescentes, suas crenças e valores, as expectativas para o futuro e a participação nos programas de intervenção promovidos pela instituição. Nas sentenças propostas sobre Drogas estavam envolvidas questões sobre risco, manutenção e proteção relativas a promoção de saúde. De uma maneira geral, os adolescentes consideraram como facilitadores para 0 abuso de drogas (risco para saúde), problemas na rede de apoio sócio-afetiva (conflitos na família, influência inadequada de amigos e vizinhança de risco) e características pessoais (baixa auto-estima, problemas de relacionamento interpessoal, insegurança afetiva). A manutenção da saúde relacionou-se com características pessoais como o uso de informação, da inteligência, da consciência e da auto-estima. Estas características evidenciam a influência da crença adolescente de controlar a própria vida e não precisar de apoio ou ajuda externa para se manter saudável. A proteção da saúde (a interrupção do uso de drogas) aparece associada ao tratamento especializado, conscientização e, em poucos casos, a uma rede de apoio eficaz. Contudo, é relevante o dado da descrença de muitos adolescentes na possibilidade de recuperação do abuso de drogas. A temática de Drogas mostrou a dinâmica adolescente na busca de responsabilização pelo que pode vir a 
acontecer em sua vida. Neste grupo, em especifico, percebeu-se a necessidade de se estimular a rede de apoio como meio de promoção e manutenção de saúde e fortalecer a busca de recursos e estratégias pessoais para evitar problemas com abuso de drogas.

As respostas das sentenças HIV/AIDS revelam que os adolescentes mostraram-se sensibilizados para a realidade de vida dos portadores do vírus HIV. Eles salientaram a importância dos soropositivos contarem com o apoio social e afetivo, eficaz e bem informado, capaz de of erecer a ajuda e o tratamento necessários. Estes dados podem ser contextualizados dentro da realidade de risco vivida por estes adolescentes cotidianamente.

As sentenças sobre sexualidade enfatizaram questões de gênero relativas ao uso do preservativo e do contraceptivo. As respostas dadas pelos adolescentes acompanham um sentido "politicamente correto", enfatizando a mulher como dona de seu próprio corpo e responsável pelo seu prazer e saúde. Ao mesmo tempo, o adolescente aparece como alguém que deve ser prevenido e conscientizado na sua iniciação sexual. De uma maneira geral, buscou-se no trabalho realizado pelo PISAD um espaço de conscientização destes adolescentes acerca de seus direitos e deveres enquanto cidadãos, e de uma retomada das suas responsabilidades enquanto guias de sua saúde e de suas vidas.

\section{CONSIDERAÇÕES FINAIS}

O trabalho com os adolescentes possibilitou a percepção de alguns aspectos marcantes tanto em nível individual como da realidade sóciocultural, que influenciam suas crenças, seus valores e suas opiniões sobre os temas abordados. Entre estes aspectos, a auto-estima, o grupo de amigos e a vivência cotidiana de risco são alguns exemplos. Em termos de promoção e prevenção em saúde ficou evidenciada a importância de desenvolver trabalhos que envolvam a possibilidade do resgate de meios de proteção como a família, a escola e a comunidade. Esta seria uma forma de restabelecer uma rede de apoio social e afetiva eficaz para esses adolescentes, considerada fator fundamental para a prevenção do uso indevido de drogas, contaminação por HIV e dificuldades em relação à sexualidade.

Este artigo, descrevendo um modelo de participação universitána na extensão comunitária, traz, também, a importância de se compreender a Psicologia enquanto uma ciência aplicada. Os pressupostos da Psicologia Comunitária, aliados aos conceitos do Modelo dos Sistemas Ecológicos, possibilitam a criação de programas de intervenção comunitários contextualizados e eficazes, valorizando a participação e a responsabilidade de cada indivíduo na construção de seu desenvolvimento, cidadania e realidade cultural.

\begin{abstract}
This article presents the Intervention Program in Sexuality, AIDS and Drugs (PISAD) from the Federal University of Rio Grande do Sul and one example of an intervention activity with adolescents at risk. The Ecological Approach to Human Development and the Community Psychology Approach are the theories used to support PISAD and they are presented with some comments about adolescent development, emphasizing risk behavior with respect to healthy sexuality, HIV/AIDS and drugs. During the intervention, a needs analysis was conducted at the beginning, and an open-ended sentences instrument was applied at the end, in order to investigate the participants' demands and concerns. Results showed that the adolescents had questions about HIV transmission and prevention, that they believed in the importance of effective social networks in promoting healthy behavior and that daily experiences influenced adolescent development, including self-esteem, identity, peer groups and sexual behavior.
\end{abstract}

Key-words: sexuality; AIDS; drugs; adolescent; intervention.

\section{REFERÊNCIAS BIBLIOGRÁFICAS}

ALVES, RB.; BRITO, R. C.; DOTTA, R. M. Programa de Intervenção em sexualidade, AIDS e drogas: Material de apoio. Manuscrito não publicado, Universidade Federal do Rio Grande do Sul, Instituto de Psicologia, Centro de Estudos Psicológicos sobre Meninos e Meninas de Rua, Porto Alegre, RS,1997.

ALVES, P.B.; BRITO, R.C.; DOTTA, R.M. Jogo de sentenças incompletas sobre sexualidade,
AIDS e drogas para adolescentes. Manuscrito não publicado, Universidade Federal do Rio Grande do Sul, Instituto de Psicologia, Centro de Estudos Psicológicos sobre Meninos e Meninas de Rua, Porto Alegre,RS, 1998.

BERNARDIS, M. A deseducacão sexual. São Paulo: Summus, 1985.

BIASOLI-ALVES, Z. M. M. Em: D. G. SOUZA, V.R.OTERO \& Z.M.M. BLASOLI-ALVES (Orgs.), Intersecções das abordagens qualitativas e quantitativas. Anais da $l^{a}$ Reunião 
Anual de Psicologia da Sociedade de Psicologia de Ribeirão Preto. Ribeirão Preto,1988. p.487-489.

BOYIER, C. B.; SHAFER M.; TSCHAN, J. M. Evaluation of a knowledge and cognitive behavioral skills Building intervention to prevent STDs and HIV infection in high school students. Adolescence, 32 (125): 25- 42, 1997.

BRITO, R.C. Uso de drogas entre meninos e meninas em situação de rua: Subsídios para uma intervenção comunitária. Porto Alegre, RS, 1999. [Dissertação de Mestrado - Programa de Pós-Graduação em Psicologia do Desenvolvimento, Universidade Federal do Rio Grande do Sul].

BRONFENBRENNER, U. The ecology of cognitive development: Research models and fugitive findings. Em: R. WOZNIAK \& K. FISCHER (Eds.), Development in Context: Acting and Thinking in Specific Environments (pp. 3-44). Hillsdale, N. J.: Erlbaum, 1993.

BRONFENBRENNER, U. Developmental ecology through space and time: A future perspective. Em: RM. MOEN; G.H. ELDER \& K. LUSCHER (Eds.). Examining lives in context (pp. 619-647). Washington: American Psychological Association, 1995.

BRONFENBRENNER, U. A Ecologia do desenvolvimento humano: experimentos naturais e planejados. Porto Alegre: Artes Médicas, 1996. (Originalmente publicado em 1979).

BRONFENBREI INER, U.; MORRIS, P. The ecology of developmental processes. Em: w. DAMON (Org.), Handbook of Child Psychology (Vol. I, pp. 9931027). New York: John Wiley \& Sons, 1998.

BRUNNER, A. B.; FISHMAN, M. Adolescent and illicit drug use. Journal of the American Medical Association, 280 (7): 597-598,1998.

CORCORAN, J.; MILLER, P.O.; BULTAM, L. Effectiveness of prevention programs for adolescent pregnancy: A meta analysis. Journal of Marriage and Family, 59: 551-567, 1997.

DICLEMENTE, R. J. Preventing sexually transmitted infection among adolescents. Journal of the American Medical Association, 279 (19): 15741575, 1998.
DUFFY, K.G.; WONG, F. Y. Community psychology. Boston: Allyn \& Bacon, 1996.

GARMEZY, N.; MASTEN, A. Chronic adversities. Em: M. RU TER; E. TAYLOR \& L. HERZOV (Orgs.), Child and adolescence psychiatry (pp.191-208). Oxford: Blackweel Scientific Publications, 1994.

GOUVÊA, D. R. Sexo, sexualidade e doenças sexualmente transmissíveis. São Paulo: Moderna, 1995.

GREEMLEY, D. G.; LEE, P. A. Condom and other contraceptive use among a random sample of female adolescents: A snapshot in time. Adolescent, 32 (128): 772-779, 1997.

KLEIN, J. D. The national longitudinal study on adolescent health. Journal of the American Medical Association, 278 (10): 864 - 865, 1997.

LEVINE, M.; PERKINS, D. V. Principies of community psychology. New York: Oxford University Press, 1987.

MACEDO, R. M. S.; SOUZA, R. M. Adolescência e sexualidade: uma proposta de educação para a família. Coletâneas da ANPEPP, 1 (2): 7-33, 1996.

MURPH, J.J.; BOGGES, S. Increased condom use among teenage males, 1988 - 1995: the role of attitudes. Family Planning Perspectives, 30 (6): 276-280, 1998.

ORFORD, J. Community psychology. theory and practive. New York: John Wiley \& Sons, 1992.

RIBEIRO, P. R. M. Educação sexual além da informação. São Paulo: EPU, 1990.

RUTTER, M. Resilience: Some conceptual considerations. Journal of Adolescent Health, 14: 626-631, 1993.

SAYÃO, R. Em: AQUINO, J.G. (Org.), Sexualidade na escola: alternativas teóricas e práticas. São Paulo: Summus, 1997.

STEINBERG, L. Adolescence. New York: Mc Graw-Hill, Inc, 1999.

WELLER, D. L. Closeness to parents helps health of teens. The Chronicle of Higher Education, September, 1997.

Recebido em 10/06/2000 Aprovado em 02/08/2000 